\title{
Análisis comparativo de los planes de estudio de filosofía en Colombia. Parte 1: generalidades y flexibilidad ${ }^{1}$
}

\author{
Comparative Analysis of Philosophy Study Plans \\ in Colombia. Part 1: Generalities and Flexibility
}

\author{
Alejandro Farieta ${ }^{2}$ \\ Marcela del Pilar Gómez ${ }^{3}$ \\ Sergio Almeida Moreno 4
}

\section{Resumen}

A continuación presentamos un análisis comparativo de los planes de estudio de filosofía en Colombia. Se abordan todos los planes de estudios en cuyo título aparece la palabra "filosofía" y se clasifican con respecto a cinco criterios: título otorgado (profesional o licenciado); tipo de institución (oficial o privada); especificidad disciplinar (filosofía o filosofía y otras disciplinas); registro ante el sNIEs (calificado o de alta calidad); modalidad (presencial o distancia). Se establecen las principales diferencias de acuerdo a tres categorías: duración en semestres, número de créditos y porcentaje de flexibilidad. Se concluye que las principales diferencias en las tres categorías se encuentran entre las licenciaturas de instituciones oficiales y las de instituciones de carácter privado.

Palabras clave

Filosofía, educación superior, plan de estudios, licenciatura en filosofía, enseñanza de la filosofía.

Abstract

This is a comparative study of the undergraduate philosophy programs in Colombia. It studies all the undergraduate programs whose title includes the expression "philosophy" and classifies it in relation to five criteria: degree (professional or educational); institution (public or private); disciplinary specificity (philosophy or philosophy and other disciplines); SNIES register (qualified or high quality); system (on-campus or distance). It establishes the main differences according to three categories: length of semesters, number of credits and percentage of flexibility. It concludes that the main differences in the three categories are between the educational programs that belongs to public institutions and those that belongs to private ones.

Key words

Philosophy, higher education, study plan, Philosophy degree, teaching philosophy.

Artículo recibido el 28 de octubre de 2014 y aprobado el 7 de abril de 2015

1 El presente estudio surgió de la necesidad de llevar a cabo una renovación del plan de estudios de la Licenciatura en Filosofía de la Universidad Pedagógica Nacional y concluyó como parte del proyecto de investigación “DCS385-14, Ética de la virtud y formación de ciudadanía" auspiciado por el Centro de Investigaciones de la Universidad Pedagógica Nacional. Agradecemos al ciup por su apoyo, a los profesores de la Licenciatura en Filosofía que conocieron parcialmente los resultados e hicieron importantes comentarios, a los monitores Natalia Morales y Fredy Gómez, quienes colaboraron en la recolección y sistematización de la información, y al evaluador anónimo de la revista por sus sugerencias para la mejora del manuscrito.

2 Universidad Pedagógica Nacional, Universidad Nacional de Colombia, Bogotá. Correo electrónico rfarieta@pedagogica.edu.co

3 Universidad Pedagógica Nacional, Universidad Nacional de Colombia, Bogotá. Correo electrónico mpgomez@pedagogica.edu.co

4 Universidad Pedagógica Nacional, Universidad Nacional de Colombia, Bogotá. Correo electrónico salmeidam@pedagogica.edu.co 


\section{Introducción}

A continuación se presenta un análisis comparativo exploratorio de los planes de estudios de los programas de pregrado que se encuentran registrados en Colombia, y en cuyo nombre se encuentra el término "filosofía". Este estudio tiene por objetivo explorar las principales diferencias de los programas de la enseñanza en filosofía en Colombia a nivel superior. También se examinan las posibles consecuencias de la implementación de los Lineamientos de calidad para las licenciaturas en educación [Lineamientos] para los programas de licenciatura en filosofía.

La comparación de los distintos programas de filosofía que presentamos a continuación se propone, en primer lugar, servir de orientación y contribución para el análisis tanto de la apertura de nuevos programas en filosofía como para las futuras renovaciones curriculares y cambios de planes de estudios que se propongan en estas áreas en las distintas universidades. En segundo lugar, es nuestro propósito con esta contribución revisar los criterios de calidad con los que se examinan los distintos programas, no con el fin de establecer una medición o una valoración, sino con el fin de contribuir a la aclaración y refinamiento de los criterios de calidad que deben seguir los planes de estudios y los aspectos en los que tales criterios se deben basar. No hemos querido - ni es nuestro interés- privilegiar una única manera de conformar un plan de estudios, pues consideramos que la calidad es una finalidad que se puede alcanzar de múltiples maneras y a través de distintos caminos.

Se obtuvo información de los planes de estudios a través de la base de datos del Sistema Nacional de Información en Educación Superior (sNies) y por consulta de cada uno de los planes de estudios. Posteriormente se analizaron sus principales diferencias de acuerdo con cinco principales categorías:

- Título otorgado

Profesional

Licenciado

- Tipo de institución

Oficial

Privada
- Especificidad disciplinar

Filosofía

Filosofía y otras (letras, humanidades, ciencias religiosas, etc.).

- Registro ante el sNiEs

Registro calificado

Registro de alta calidad

- Modalidad

\section{Presencial}

Distancia (distancia tradicional o virtual). 5

En un segundo momento, el estudio se realizó comparando el número de créditos que cada programa incluye como créditos flexibles. Dado que la principal distinción entre los programas tiene que ver con el título otorgado, se analizaron todos los demás factores de análisis con respecto a la distinción entre los programas de estudios profesionales en filosofía y de licenciatura en filosofía.

Para concluir, mostraremos cómo la propuesta de Lineamientos podrá incidir en los programas de licenciatura en filosofía, en particular en las siguientes características específicas que todo programa deberá cumplir: todas las licenciaturas deberán tener una duración de cinco años y ser presenciales (Ministerio de Educación Nacional, MEN, 2014, p. 33).

\section{Metodología}

La metodología utilizada en esta investigación fue la del estudio comparativo. De acuerdo con la información que se encuentra en la base de datos del sNies, en la actualidad están registrados y activos cincuenta y siete programas de pregrado con el término "filosofía" en su nombre; hemos tomado para el presente estudio cincuenta y cuatro programas (tabla 1).

5 Aunque hay dos modalidades de programas académicos de filosofía en Colombia, de acuerdo con la información que ofrece el SNIES (distancia tradicional y virtual), estas dos modalidades se han agrupado en un solo ítem, puesto que solamente hay un programa virtual: el programa de Licenciatura en Filosofía y Educación Religiosa de la Fundación Universitaria Católica del Norte. 
Tabla 1. Programas de pregrado en filosofía activos en Colombia ${ }^{6}$

\begin{tabular}{|c|c|c|c|c|c|c|}
\hline $\begin{array}{l}\text { Cód. } \\
\text { SNIES }\end{array}$ & Nombre de la institución & Nombre del programa & Créd. & Sem. & Modalidad & Municipio (Depto.) \\
\hline 1300 & $\begin{array}{l}\text { Colegio Mayor de Nuestra } \\
\text { Señora del Rosario }\end{array}$ & Filosofía & 144 & 8 & Presencial & Bogotá D. C. \\
\hline 2049 & $\begin{array}{l}\text { Corporación Universitaria } \\
\text { Minuto de Dios }\end{array}$ & Licenciatura en Filosofía & 144 & 8 & Presencial & Bogotá D. C. \\
\hline 9363 & $\begin{array}{l}\text { Corporación Universitaria } \\
\text { Minuto de Dios }\end{array}$ & Estudios en Filosofía & 144 & 8 & Presencial & Bogotá D. C. \\
\hline 53355 & $\begin{array}{l}\text { Fundación Universidad } \\
\text { Autónoma de Colombia }\end{array}$ & Filosofía & 128 & 8 & Presencial & Bogotá D. C. \\
\hline 9570 & $\begin{array}{l}\text { Fundación Universitaria } \\
\text { Católica del Norte }\end{array}$ & $\begin{array}{l}\text { Licenciatura en Filosofía } \\
\text { y Educación Religiosa }\end{array}$ & 139 & 8 & Virtual & $\begin{array}{l}\text { Santa Rosa de } \\
\text { Osos (Antioquia) }\end{array}$ \\
\hline 4378 & $\begin{array}{l}\text { Fundación Universitaria } \\
\text { Católica Lumen Gentium }\end{array}$ & $\begin{array}{l}\text { Licenciatura en Filosofía } \\
\text { y Ciencias Religiosas }\end{array}$ & 157 & 10 & Presencial & $\begin{array}{l}\text { Cali (Valle del } \\
\text { Cauca) }\end{array}$ \\
\hline 20444 & $\begin{array}{l}\text { Fundación Universitaria } \\
\text { Luis Amigó }\end{array}$ & Licenciatura en Filosofía & 143 & 9 & Presencial & Medellín (Antioquia) \\
\hline 20650 & $\begin{array}{l}\text { Fundación Universitaria } \\
\text { Luis Amigó }\end{array}$ & Licenciatura en Filosofía & 143 & 9 & $\begin{array}{l}\text { Distancia } \\
\text { (tradicional) }\end{array}$ & Medellín (Antioquia) \\
\hline 53758 & $\begin{array}{l}\text { Fundación Universitaria } \\
\text { Luis Amigó }\end{array}$ & Filosofía & 142 & 8 & $\begin{array}{l}\text { Distancia } \\
\text { (tradicional) }\end{array}$ & Medellín (Antioquia) \\
\hline 11971 & $\begin{array}{l}\text { Fundación Universitaria } \\
\text { Luis Amigó }\end{array}$ & Filosofía & 142 & 8 & Presencial & Medellín (Antioquia) \\
\hline 54345 & $\begin{array}{l}\text { Fundación Universitaria } \\
\text { San Alfonso }\end{array}$ & Licenciatura en Filosofía & 128 & 8 & Presencial & Bogotá D. C. \\
\hline 11929 & $\begin{array}{l}\text { Fundación Universitaria } \\
\text { San Alfonso }\end{array}$ & Filosofía Teología & 166 & 10 & Presencial & Bogotá D. C. \\
\hline 933 & $\begin{array}{l}\text { Pontificia Universidad } \\
\text { Javeriana }\end{array}$ & Licenciatura en Filosofía & 186 & 10 & Presencial & Bogotá D. C. \\
\hline 955 & $\begin{array}{l}\text { Pontificia Universidad } \\
\text { Javeriana }\end{array}$ & Filosofía & 150 & 9 & Presencial & Bogotá D. C. \\
\hline 54163 & $\begin{array}{l}\text { Pontificia Universidad } \\
\text { Javeriana }\end{array}$ & Filosofía & 148 & 8 & Presencial & $\begin{array}{l}\text { Cali (Valle del } \\
\text { Cauca) }\end{array}$ \\
\hline 417 & Universidad de Antioquia & Licenciatura en Filosofía & 200 & 10 & Presencial & Medellín (Antioquia) \\
\hline 90553 & Universidad de Antioquia & Licenciatura en Filosofía & 200 & 10 & Presencial & $\begin{array}{l}\text { Carmen de Viboral } \\
\text { (Antioquia) }\end{array}$ \\
\hline 91267 & Universidad de Antioquia & Licenciatura en Filosofía & 170 & 10 & Presencial & Caucasia (Antioquia) \\
\hline 441 & Universidad de Antioquia & Filosofía & 172 & 10 & Presencial & Medellín (Antioquia) \\
\hline 13218 & Universidad de Caldas & $\begin{array}{l}\text { Licenciatura en } \\
\text { Filosofía y Letras }\end{array}$ & 161 & 10 & Presencial & Manizales (Caldas) \\
\hline 295 & Universidad de Caldas & Filosofía y Letras & 161 & 10 & Presencial & Manizales (Caldas) \\
\hline
\end{tabular}

6 Debido a que no fue brindada información de parte de la institución, ni se encontró información precisa de los planes de estudios, no se han tenido en cuenta los siguientes programas: (1) Licenciatura en Filosofía y Educación Religiosa de la Universidad Católica de Oriente (Rionegro, Ant.); (2) Filosofía de la Universidad de Ibagué (Tolima); (3) Licenciatura en Filosofía y Estudios Políticos de la Universidad del Magdalena. 
Universidad Pedagógica Nacional

Facultad de Humanidades

\begin{tabular}{|c|c|c|c|c|c|c|}
\hline $\begin{array}{l}\text { Cód. } \\
\text { SNIES }\end{array}$ & Nombre de la institución & Nombre del programa & Créd. & Sem. & Modalidad & Municipio (Depto.) \\
\hline 744 & Universidad de Cartagena & Filosofía & 160 & 10 & Presencial & Cartagena (Bolívar) \\
\hline 90806 & Universidad de la Sabana & Filosofía & 163 & 9 & Presencial & $\begin{array}{l}\text { Chía } \\
\text { (Cundinamarca) }\end{array}$ \\
\hline 1445 & Universidad de la Salle & Filosofía y Letras & 155 & 8 & Presencial & Bogotá D. C. \\
\hline 1537 & Universidad de los Andes & Filosofía & 123 & 8 & Presencial & Bogotá D. C. \\
\hline 2715 & Universidad de Nariño & $\begin{array}{l}\text { Licenciatura en } \\
\text { Filosofía y Letras }\end{array}$ & 170 & 10 & Presencial & Pasto (Nariño) \\
\hline 11927 & Universidad de Pamplona & Filosofía & 156 & 10 & Presencial & $\begin{array}{l}\text { Pamplona (Norte } \\
\text { de Santander) }\end{array}$ \\
\hline 1371 & $\begin{array}{l}\text { Universidad de San } \\
\text { Buenaventura }\end{array}$ & Licenciatura en Filosofía & 137 & 8 & Presencial & Bogotá D. C. \\
\hline 9693 & Universidad del Atlántico & Filosofía & 136 & 8 & Presencial & $\begin{array}{l}\text { Barranquilla } \\
\text { (Atlántico) }\end{array}$ \\
\hline 11967 & Universidad del Cauca & Filosofía & 132 & 8 & Presencial & Popayán (Cauca) \\
\hline 14826 & Universidad del Quindío & Filosofía & 143 & 10 & Presencial & Armenia (Quindío) \\
\hline 557 & Universidad del Valle & Licenciatura en Filosofía & 149 & 10 & Presencial & $\begin{array}{l}\text { Cali (Valle del } \\
\text { Cauca) }\end{array}$ \\
\hline 11374 & Universidad del Valle & Profesional en Filosofía & 123 & 8 & Presencial & $\begin{array}{l}\text { Cali (Valle del } \\
\text { Cauca) }\end{array}$ \\
\hline 53049 & Universidad El Bosque & Filosofía & 144 & 8 & Presencial & Bogotá D. C. \\
\hline 12047 & $\begin{array}{l}\text { Universidad Externado } \\
\text { de Colombia }\end{array}$ & Filosofía & 170 & 10 & Presencial & Bogotá D. C. \\
\hline 8460 & $\begin{array}{l}\text { Universidad Industrial } \\
\text { de Santander }\end{array}$ & Filosofía & 135 & 8 & Presencial & $\begin{array}{l}\text { Bucaramanga } \\
\text { (Santander) }\end{array}$ \\
\hline 91044 & Universidad La Gran Colombia & $\begin{array}{l}\text { Licenciatura en } \\
\text { Filosofía e Historia }\end{array}$ & 155 & 10 & Presencial & Bogotá D. C. \\
\hline 8167 & Universidad Libre & Filosofía & 155 & 10 & Presencial & Bogotá D. C. \\
\hline 4450 & $\begin{array}{l}\text { Universidad Nacional } \\
\text { Abierta y a Distancia }\end{array}$ & Licenciatura en Filosofía & 160 & 10 & $\begin{array}{l}\text { Distancia } \\
\text { (tradicional) }\end{array}$ & Bogotá D. C. \\
\hline 4074 & $\begin{array}{l}\text { Universidad Nacional } \\
\text { Abierta y a Distancia }\end{array}$ & Filosofía & 160 & 8 & $\begin{array}{l}\text { Distancia } \\
\text { (tradicional) }\end{array}$ & Bogotá D. C. \\
\hline 20 & $\begin{array}{l}\text { Universidad Nacional } \\
\text { de Colombia }\end{array}$ & Filosofía & 122 & 8 & Presencial & Bogotá D. C. \\
\hline 53718 & $\begin{array}{l}\text { Universidad Pedagógica } \\
\text { Nacional }\end{array}$ & Licenciatura en Filosofía & 160 & 10 & Presencial & Bogotá D. C. \\
\hline 10463 & $\begin{array}{l}\text { Universidad Pedagógica y } \\
\text { Tecnológica de Colombia }\end{array}$ & Licenciatura en Filosofía & 175 & 10 & Presencial & Tunja (Boyacá) \\
\hline 2718 & $\begin{array}{l}\text { Universidad Pontificia } \\
\text { Bolivariana }\end{array}$ & $\begin{array}{l}\text { Licenciatura en } \\
\text { Filosofía y Letras }\end{array}$ & 160 & 10 & Presencial & Medellín (Antioquia) \\
\hline 1198 & $\begin{array}{l}\text { Universidad Pontificia } \\
\text { Bolivariana }\end{array}$ & Filosofía y Letras & 128 & 8 & Presencial & Medellín (Antioquia) \\
\hline
\end{tabular}




\begin{tabular}{|c|c|c|c|c|c|c|}
\hline $\begin{array}{l}\text { Cód. } \\
\text { SNIIES }\end{array}$ & Nombre de la institución & Nombre del programa & Créd. & Sem. & Modalidad & Municipio (Depto.) \\
\hline 1197 & $\begin{array}{l}\text { Universidad Pontificia } \\
\text { Bolivariana }\end{array}$ & Filosofía & 125 & 8 & Presencial & Medellín (Antioquia) \\
\hline 10798 & Universidad Santo Tomás & $\begin{array}{l}\text { Licenciatura en Filosofía, } \\
\text { Ética y Valores Humanos }\end{array}$ & 157 & 10 & $\begin{array}{l}\text { Distancia } \\
\text { (tradicional) }\end{array}$ & Bogotá D. C. \\
\hline 13217 & Universidad Santo Tomás & $\begin{array}{l}\text { Licenciatura en Filosofía } \\
\text { y Lengua Castellana }\end{array}$ & 158 & 8 & Presencial & Bogotá D. C. \\
\hline 1077 & Universidad Santo Tomás & $\begin{array}{l}\text { Licenciatura en Filosofía } \\
\text { y Educación Religiosa }\end{array}$ & 157 & 10 & $\begin{array}{l}\text { Distancia } \\
\text { (tradicional) }\end{array}$ & Bogotá D. C. \\
\hline 10793 & Universidad Santo Tomás & $\begin{array}{l}\text { Licenciatura en } \\
\text { Filosofía, Pensamiento } \\
\text { Político y Económico }\end{array}$ & 157 & 10 & $\begin{array}{l}\text { Distancia } \\
\text { (tradicional) }\end{array}$ & Bogotá D. C. \\
\hline 773 & Universidad Sergio Arboleda & $\begin{array}{l}\text { Licenciatura en Filosofía } \\
\text { y Humanidades }\end{array}$ & 138 & 8 & Presencial & Bogotá D. C. \\
\hline 1872 & Universidad Sergio Arboleda & Filosofía y Humanidades & 133 & 8 & Presencial & Bogotá D. C. \\
\hline 10264 & $\begin{array}{l}\text { Universidad Tecnológica } \\
\text { de Pereira }\end{array}$ & Licenciatura en Filosofía & 164 & 12 & Presencial & Pereira (Risaralda) \\
\hline 90479 & Universitaria Agustiniana & Licenciatura en Filosofía & 150 & 9 & Presencial & Bogotá D. C. \\
\hline
\end{tabular}

Fuente: snies. Recuperado de: http://snies.mineducacion.gov.co/consultasnies/ programa/buscar.jsp?control=0.23816498138523357

En la actualidad, en Colombia hay dos modalidades de estudios profesionales universitarios que llevan por nombre "Filosofía". Se trata, por una parte, de los estudios profesionales en filosofía, y por otra parte las licenciaturas en filosofía. Estas dos modalidades tienen ciertas diferencias importantes, en particular porque, tal como es estipulado por la Ley 30 de 1992, "Los programas de pregrado en educación pueden conducir al título de 'Licenciado en..." (art. 25). De acuerdo con la clasificación del snies, todos los programas de estudios profesionales en filosofía se encuentran en el área de conocimiento de las ciencias sociales y humanas; mientras que todas las licenciaturas en filosofía se encuentran en el área de las ciencias de la educación. Sin embargo, se han tomado en cuenta tanto los estudios profesionales como las licenciaturas debido a que en diez de las treinta y ocho instituciones de educación superior tomadas en el estudio (26,31\%) se encuentran ambos programas y además comparten bloques importantes de espacios académicos. Los programas fueron clasificados inicialmente de acuerdo a las siguientes características (tabla 2).
Tabla 2. Número de programas de acuerdo a las características examinadas

\begin{tabular}{|l|l|l|}
\hline & & \multicolumn{1}{|c|}{$\mathbf{N}^{\mathbf{0}}$} \\
\hline programas
\end{tabular}


Gráfica 1. Número de programas de acuerdo a las características examinadas

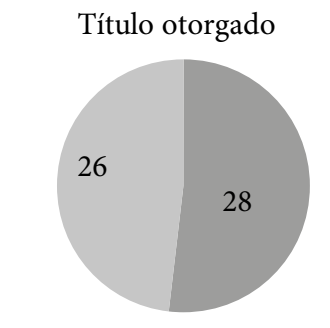

- Profesional $\Perp$ Licenciado

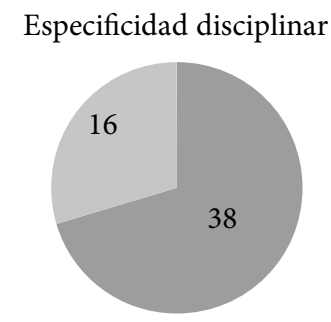

-Filosofía $\backsim$ Filosofía y otro
Registro ante el SNIES

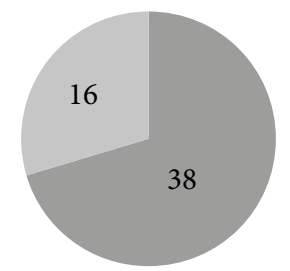

$\llbracket$ R. calificado $₫$ R. alta calidad

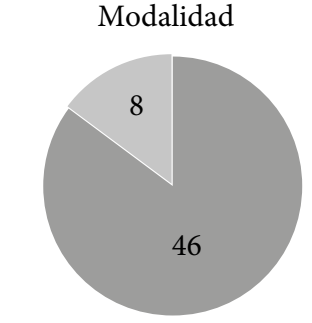

-Presencial $\backsim$ Distancia

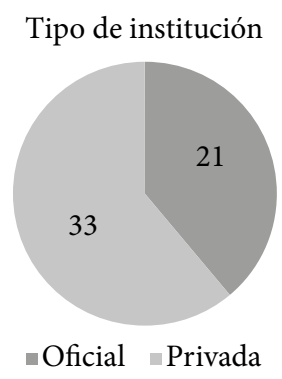

ఐOficial $\backsim$ Privada

Fuente: Elaboración propia.

Para el análisis se utilizaron las siguientes categorías de comparación de los planes de estudio:

1. Duración (semestres): la duración de cada programa se determina en número de semestres en el plan de estudios. Debido a que se registra una variación de entre ocho y doce semestres en los distintos programas - diferencia equivalente al $50 \%$ de la duración del programa-, se ha considerado un factor relevante para determinar diferencias considerables entre los distintos programas.

2. N. ${ }^{\circ}$ de créditos: el crédito es la unidad de medida de cada espacio académico que compone un plan de estudios de un programa de educación superior. Según el artículo 18 del Decreto 2566 de 2003 del MEN, un crédito equivale a 48 horas al semestre ( 3 horas semanales por crédito). Los programas oscilan entre 122 y 200 créditos, lo que representa una diferencia del $78 \%$ de la duración del programa, por lo que se considera un factor relevante para el análisis.

3. Flexibilidad: se mide en términos del porcentaje de créditos optativos del plan de estu- dios en comparación con el número total de créditos. A su vez, esta se distribuye en dos componentes:

a. Componente flexible disciplinar: consta del número de créditos que tiene cada programa que son optativos para los estudiantes, pero cuyos espacios académicos optativos se ofrecen exclusivamente para el programa. Por lo general, se trata de optativas o de líneas de profundización de los planes de estudios y dependen de la especificidad disciplinar de cada programa.

b. Componente flexible de libre elección: se refiere al número de créditos en espacios académicos de libre elección del estudiante, los cuales por lo general se presentan como electivas de contexto, electivas institucionales o electivas de carácter cultural e interdisciplinario que permiten complementar la formación de los estudiantes más allá de los espacios académicos propios del plan de estudios, acercándolo a otras disciplinas o espacios de desarrollo cultural, personal o deportivo. 


\section{Resultados}

A continuación se presenta el resultado de comparar, de acuerdo con las categorías anteriormente enunciadas, a los cincuenta y cuatro programas de pregrado en filosofía actualmente activos en Colombia. Presentaremos, en primer lugar, los resultados de acuerdo con las características generales de cada uno de los programas, su duración y número de créditos. En segundo lugar, presentaremos los resultados con respecto a la flexibilidad de cada uno de los programas, tanto en términos de número de créditos del programa como de porcentaje con respecto al número total de créditos del programa.

\section{Características generales}

\section{Duración}

La duración de los programas en filosofía varía de 8 a 12 semestres, con una media general de 9,06 y una desviación estándar de 1,04. A continuación (gráfica 2) presentamos las diferencias por programas, de acuerdo a las categorías de análisis estipuladas, y señalaremos las principales diferencias entre los programas de acuerdo con la clasificación, siempre y cuando esta diferencia supere la mitad de la desviación estándar general $(0,52)$, en cuyo caso lo señalaremos como una diferencia relevante entre los programas.

Gráfica 2. Media de duración de los programas de filosofía

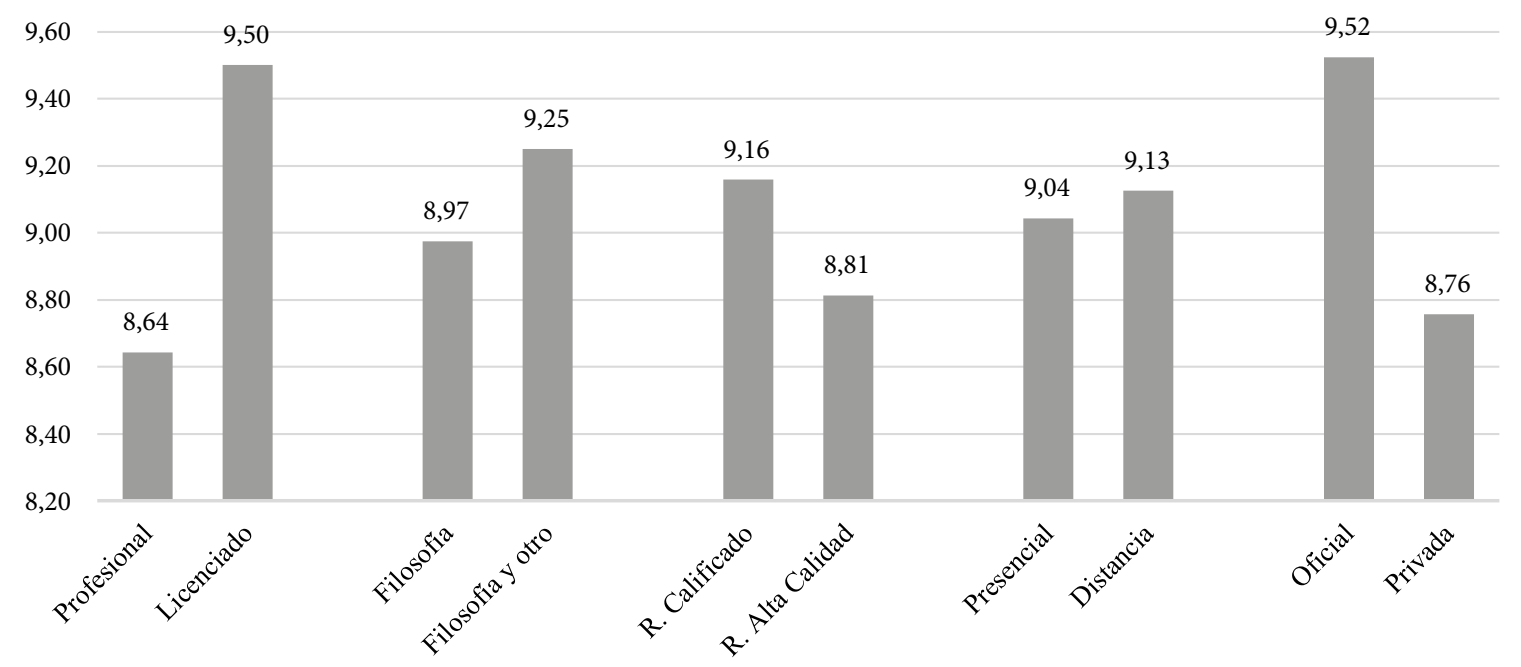

Fuente: Elaboración propia.

Una primera diferencia relevante se presenta con respecto al título otorgado: las licenciaturas tienen una duración promedio de 9,50 y los estudios profesionales 8,64 , lo que arroja una diferencia de 0,86 . También hay una diferencia relevante por tipo de institución; las instituciones oficiales tienen una media de 9,52, mientras que las privadas de 8,76, lo que arroja una diferencia de 0,76 . Vale la pena resaltar que la media de duración de las licencia- turas se encuentra 0,5 semestres por debajo de la propuesta de los Lineamientos, lo que implicaría un cambio considerable en la mayoría de las licenciaturas en filosofía.

A continuación (gráficas 3 y 4) mostraremos las medias de duración de los programas de filosofía agrupados por título otorgado y examinaremos sus principales diferencias de acuerdo con los demás criterios de clasificación de los programas. 
Universidad Pedagógica Nacional

Facultad de Humanidades

Gráfica 3. Media de duración de los programas profesionales en filosofía

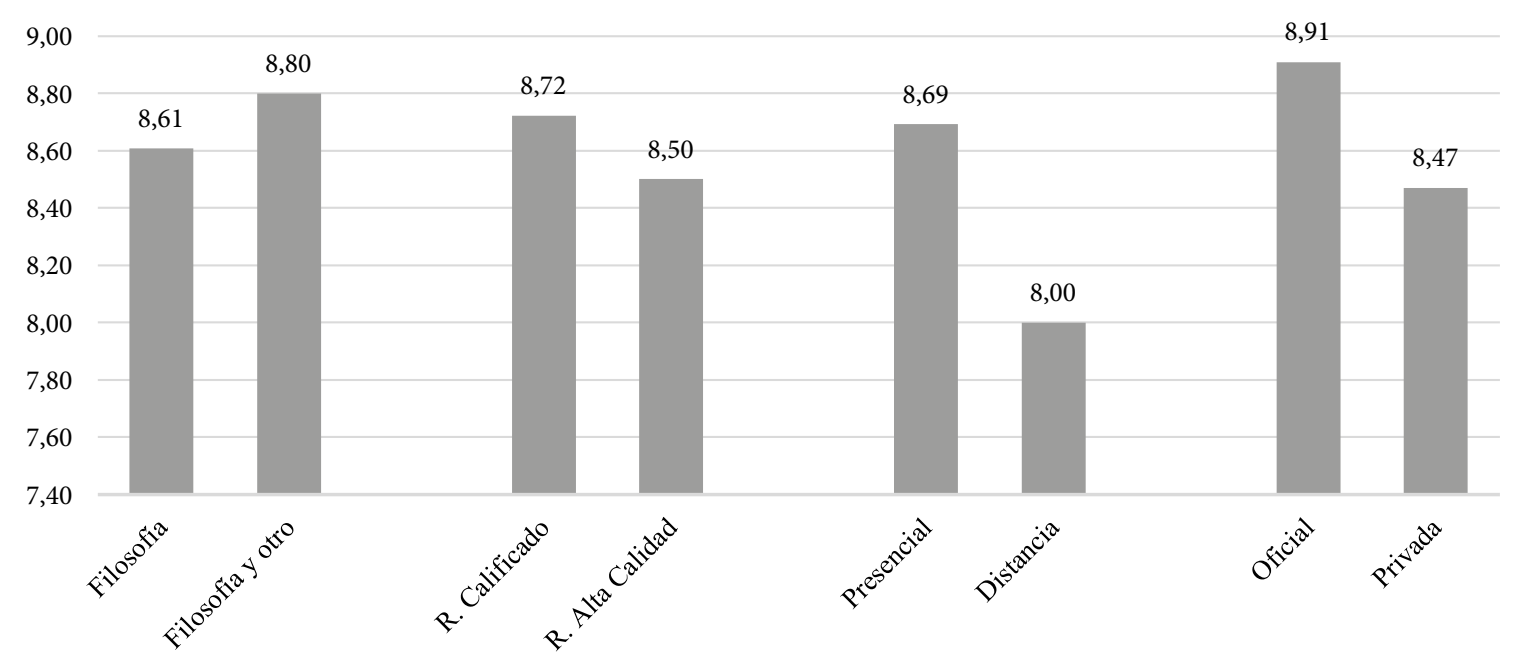

Fuente: Elaboración propia.

Con respecto a los programas profesionales en filosofía, una diferencia relevante se encuentra entre los programas presenciales, con media de 8,69, y los programas a distancia, con una media de $8 \mathrm{y}$ una diferencia de 0,69 . También hay una diferen- cia relevante entre los programas de instituciones oficiales, con una media de 8,91 y los programas de instituciones privadas, con una media de 8,47 y una diferencia de 0,44 . Las demás diferencias no son considerables.

Gráfica 4. Media de duración de los programas de licenciatura en filosofía

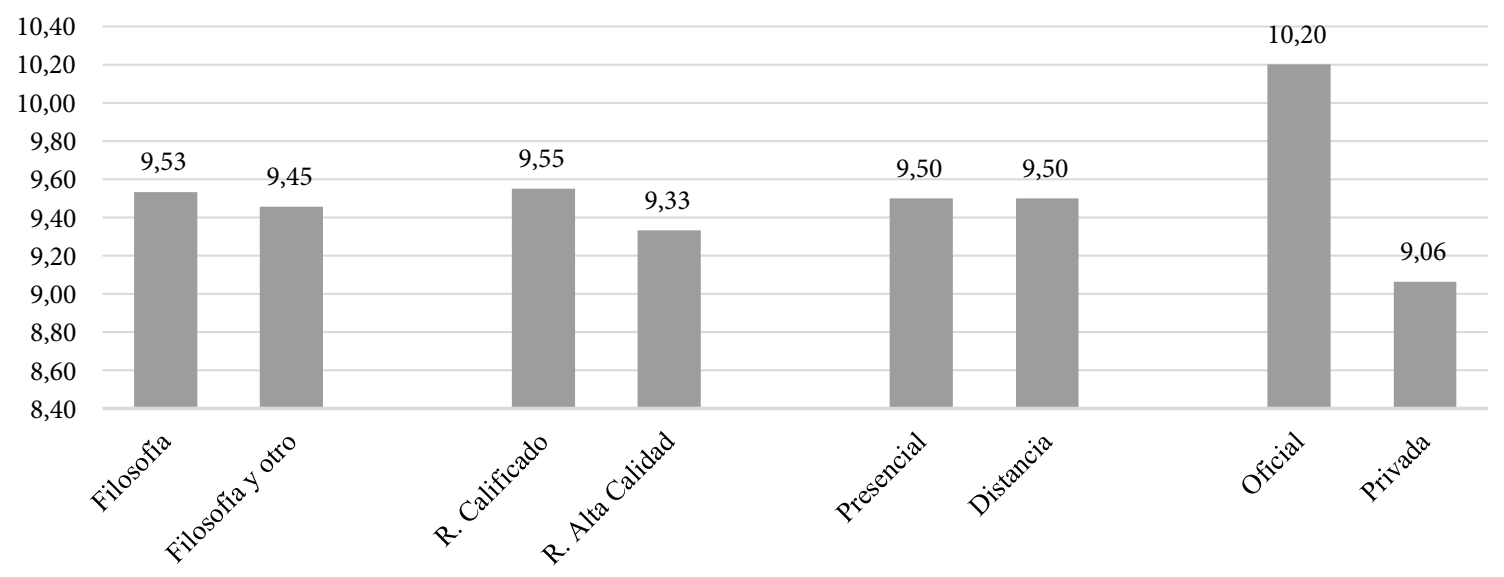

Fuente: Elaboración propia.

Con respecto a los programas de licenciatura en filosofía, la mayor y única diferencia relevante se presenta de acuerdo al tipo de institución. Las instituciones oficiales presentan un promedio de 10,20, mientras que las privadas un promedio de 9,06 , con una diferencia de 1,14. Esta es la diferencia relevante hallada en esta parte del estudio con mayor valor, y la que representaría la mayor diferencia entre los programas de filosofía. Valga la pena mencionar que, de acuerdo con la propuesta de Lineamientos, 
se verán principalmente afectados los programas de instituciones privadas. Todos los programas de universidades oficiales cumplen con el requisito de diez semestres, mientras que nueve de los dieciséis programas de licenciatura de instituciones privadas se encuentran por debajo de los diez semestres.

\section{Número de créditos}

La media general de número de créditos de los 54 programas es de 151,44 con una variación de 122 a 200 créditos y una desviación estándar de 17,61. A continuación (gráfica 5) presentaremos las diferencias por programas de acuerdo con las clasificaciones estipuladas y señalaremos las principales diferencias entre los programas de acuerdo con la clasificación, siempre y cuando esta diferencia supere la mitad de la desviación estándar general $(8,82)$, en cuyo caso lo señalaremos como una diferencia relevante entre los programas.

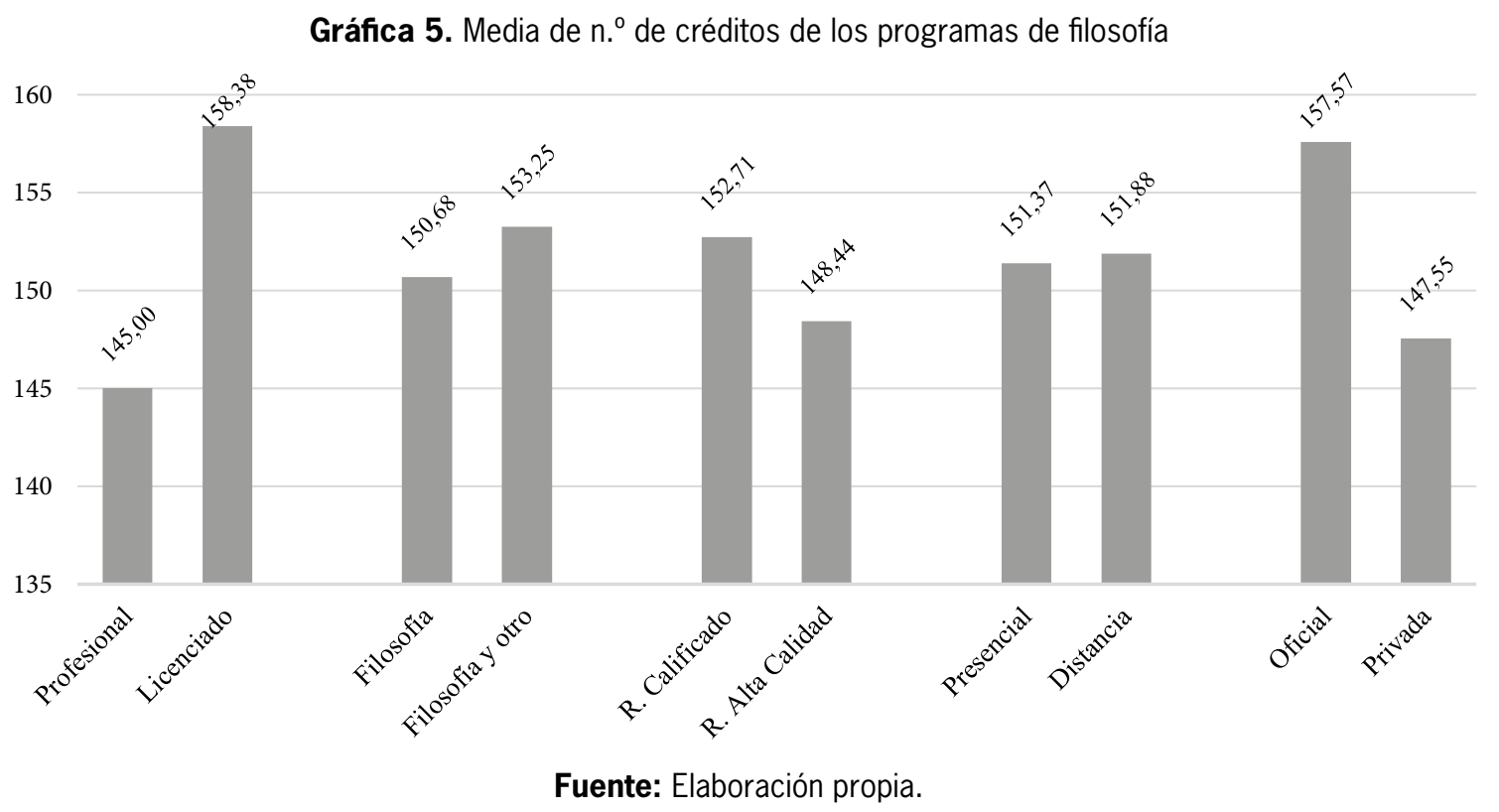

La mayor diferencia relevante entre los programas se encuentra de acuerdo al tipo de programa. Los programas profesionales tienen un promedio de 145 créditos, mientras que en las licenciaturas el promedio es de 158,38 créditos, con una diferencia de 13,38 . Otra diferencia relevante se presenta de acuerdo con el tipo de institución que ofrece el programa: 157,57 en las instituciones oficiales y 147,55 en las privadas y una diferencia de 10,02. Estas dos diferencias concuerdan con las diferencias de los programas en términos del número de semestres (cf. § 3.1.1).

A continuación (gráficas 6 y 7) mostraremos las medias de número de créditos de los programas de filosofía agrupados por título otorgado y examinaremos sus principales diferencias de acuerdo con los demás criterios de clasificación de los programas; además examinaremos si las diferencias se corresponden con las diferencias en número de semestres del programa. 
Universidad Pedagógica Nacional

Facultad de Humanidades

Gráfica 6. Media del número de créditos de los programas profesionales en filosofía

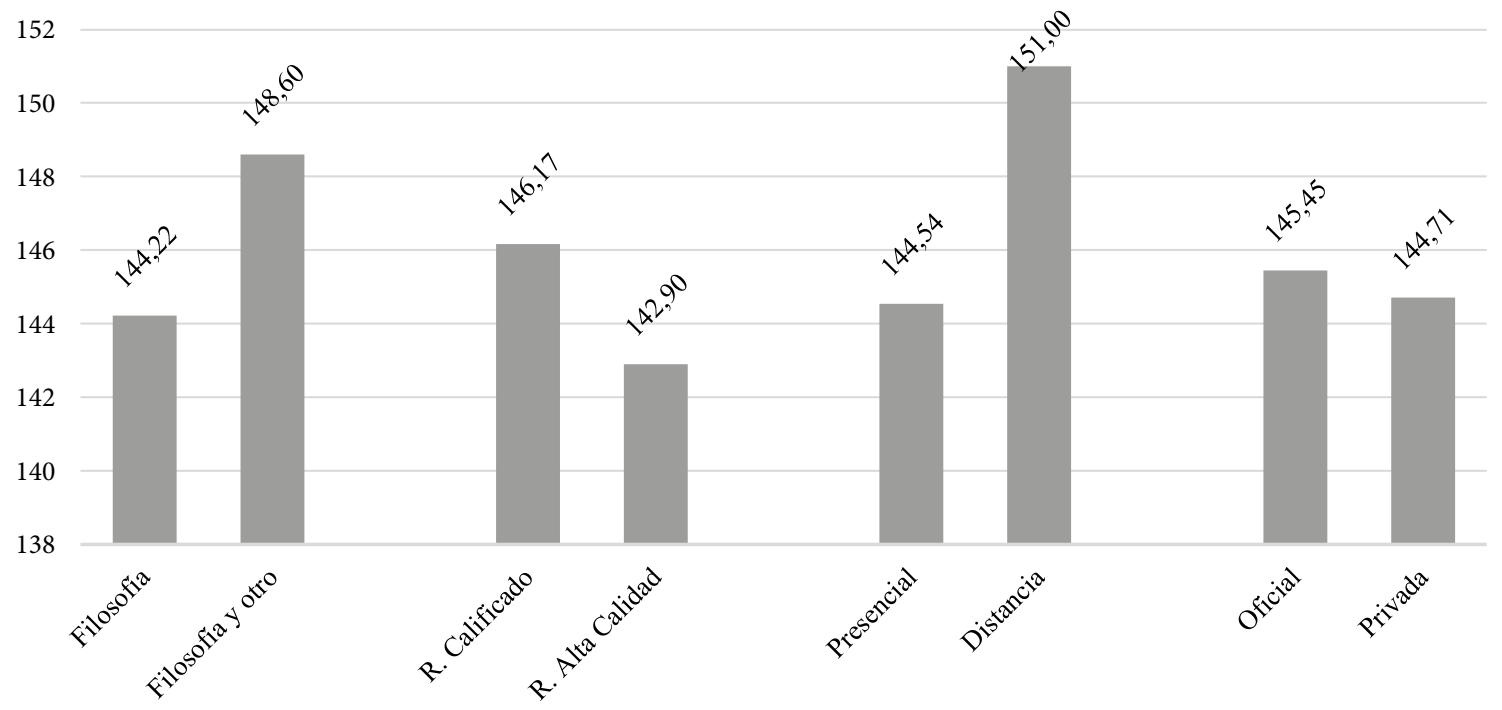

Fuente: Elaboración propia.

De acuerdo al número de créditos de los programas profesionales en filosofía, la única diferencia se presenta con relación a la modalidad del programa: los programas presenciales tienen un promedio de 144,45 mientras que los a distancia, 151, con una diferencia de 6,55 . No obstante, esta diferencia se encuentra por debajo de la mitad de la desviación estándar general y puede desestimarse. Es de notar que, con respecto a la duración del programa en semestres, se encuentran menos diferencias en el número de créditos de los programas profesionales.

Gráfica 7. Media de $n^{0}$ de créditos de los programas de licenciatura en filosofía

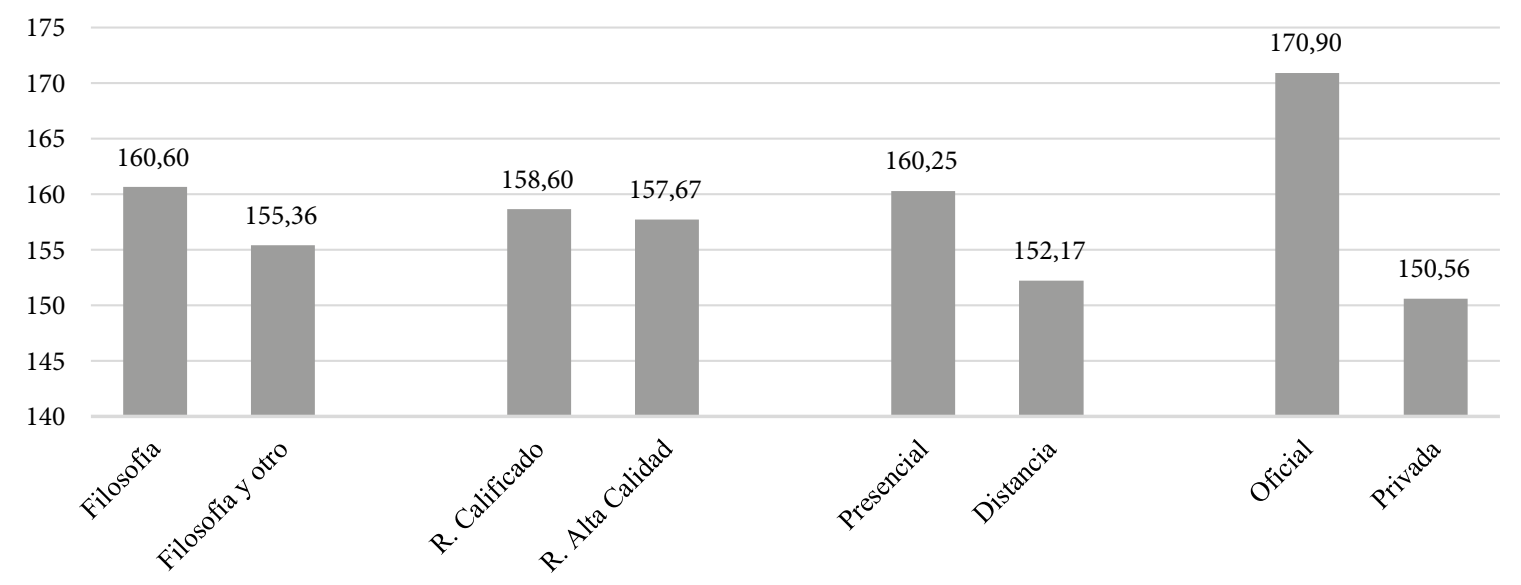

Fuente: Elaboración propia.

En las licenciaturas en filosofía, la única diferencia relevante se encuentra con respecto a la institución: los programas de instituciones oficiales tienen un promedio de 170,9 créditos, mientras que las instituciones privadas presentan un promedio de 150,56 con una diferencia de 20,34. Esta diferen- cia se corresponde con las diferencias en términos del número de semestres de los programas (cf. $₫$ 3.1.1) y es la mayor registrada entre los programas de filosofía.

De acuerdo con las características de calidad para los programas de educación que se proponen en los 
Lineamientos, estas diferencias tanto en número de créditos como en semestres podrían evidenciar una diferencia significativa en la calidad de la formación de los licenciados en filosofía, en particular, entre los programas de universidades oficiales en contraste con los programas de universidades privadas. En un estudio posterior presentaremos con detalle, en términos de estructura curricular, qué otros aspectos puntuales de los programas presentan diferencias considerables y de qué manera estas diferencias podría evidenciar diferencias de calidad entre los distintos tipos de programas.

\section{Flexibilidad}

A continuación (tabla 3 y gráfica 8 ) se presenta el análisis comparativo del porcentaje de flexibilidad de los distintos tipos de programa, de acuerdo con las clasificaciones enunciadas en la sección introductoria $(\$ 1)$.

Tabla 3. Porcentaje promedio de flexibilidad de todos los programas

\begin{tabular}{|l|l|c|c|c|c|}
\hline & & $\begin{array}{c}\text { N. } \\
\text { programas }\end{array}$ & $\begin{array}{c}\text { \% flexibilidad } \\
\text { disciplinar }\end{array}$ & $\begin{array}{c}\text { \% libre } \\
\text { elección }\end{array}$ & Flexibilidad \\
\hline Promedio general & & 54 & $18,58 \%$ & $6,73 \%$ & $25,31 \%$ \\
\hline \multirow{2}{*}{ Título otorgado } & Profesional & 28 & $20,07 \%$ & $7,72 \%$ & $27,80 \%$ \\
\hline Especificidad discipli- & Licenciado & 26 & $16,98 \%$ & $5,65 \%$ & $22,63 \%$ \\
\hline nar & Filosofía & 38 & $22,40 \%$ & $7,43 \%$ & $29,83 \%$ \\
\hline \multirow{2}{*}{ Registro ante el sNES } & Filosofía y otro & 16 & $9,51 \%$ & $5,06 \%$ & $14,57 \%$ \\
\hline \multirow{2}{*}{ Modalidad } & Registro calificado & 38 & $15,76 \%$ & $7,01 \%$ & $22,77 \%$ \\
\cline { 2 - 6 } & Registro alta calidad & 16 & $25,27 \%$ & $6,06 \%$ & $31,33 \%$ \\
\hline \multirow{2}{*}{ Tipo de institución } & Presencial & 46 & $21,63 \%$ & $5,85 \%$ & $27,48 \%$ \\
\hline & Distancia & 8 & $1,04 \%$ & $11,78 \%$ & $12,82 \%$ \\
\hline & Oficial & 21 & $32,49 \%$ & $7,82 \%$ & $40,31 \%$ \\
\hline
\end{tabular}

Gráfica 8. Porcentaje promedio de flexibilidad de todos los programas

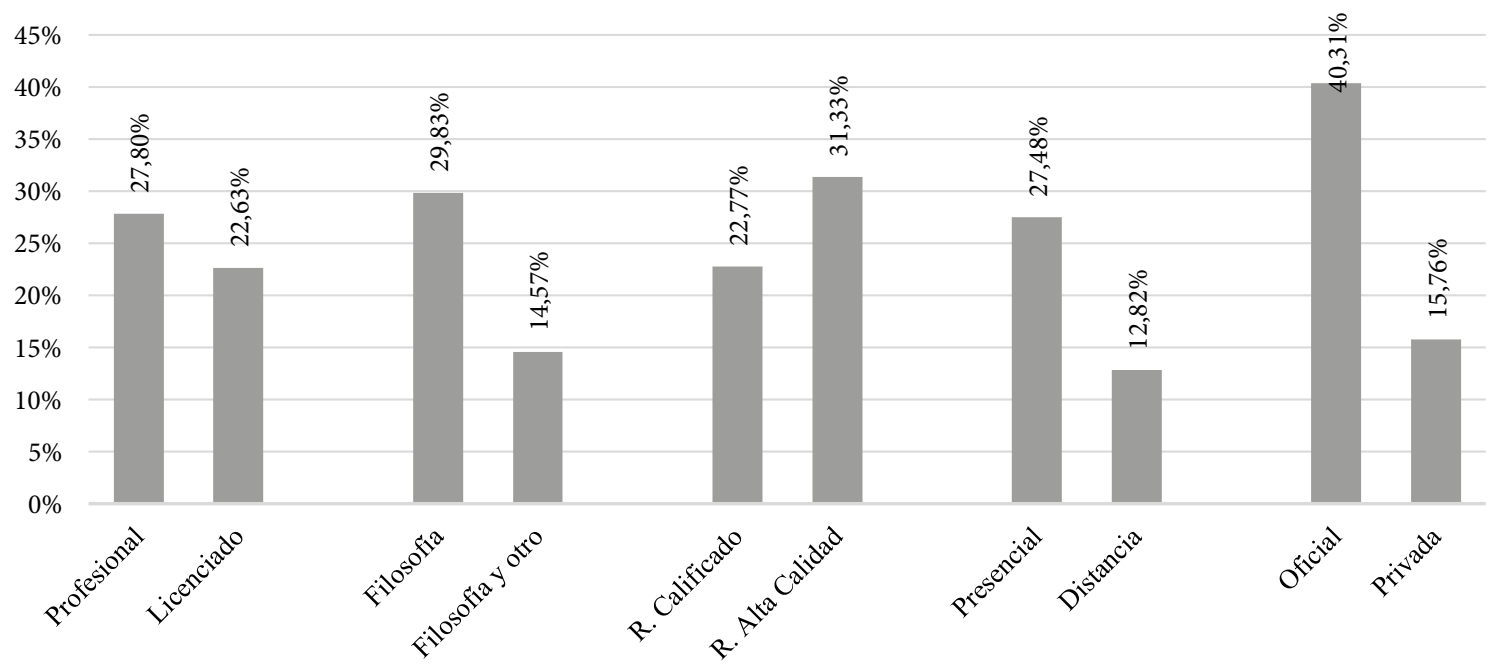

Fuente: Elaboración propia. 
Como muestra la tabla 3, hay una variación considerable del porcentaje de flexibilidad de los programas de acuerdo con el tipo de programa. Así, los estudios profesionales ofrecen un porcentaje flexible de créditos en sus programas superior al de los programas de licenciatura (27,80\%-22,63\%). Sin embargo, es importante hacer notar que, pese a que la diferencia aparentemente mayor entre los programas es la diferencia entre el título otorgado (licenciado $v s$. profesional), en términos de flexibilidad la diferencia es baja $(5,16 \%)$ y todas las otras clasificaciones ofrecen una diferencia mayor.

Con respecto a la especificidad disciplinar, son considerablemente más flexibles los programas dedicados principalmente a la filosofía $(29,83 \%)$, que los que combinan la filosofía con algún otra disciplina $(14,57 \%)$ con una diferencia relevante del $15,26 \%$; la flexibilidad de los programas dedicados exclusivamente a la filosofía ofrecen en promedio más del doble de créditos flexibles.

También es relevante la diferencia de flexibilidad entre los programas con acreditación de alta calidad $(31,31 \%)$, con respecto a los programas que solamente tienen registro calificado (22,77\%). Esta diferencia responde a los Lineamientos para la acreditación de programas de pregrado (CNA, 2013, pp. 30-31), en los que el índice de flexibilidad curricular - que aquí exponemos en términos de porcentajes - es un criterio evaluado en el momento de la acreditación.

Hay diferencia importante, también, entre el porcentaje de flexibilidad de acuerdo con su modalidad. Los programas presenciales tienen un promedio de $27,48 \%$, mientras que los programas a distancia tienen un $12,82 \%$, por lo que hay una diferencia del $14,66 \%$. Es importante resaltar que en el porcentaje de créditos flexibles disciplinares la diferencia es todavía mayor: los programas presenciales tienen un porcentaje de flexibilidad disciplinar del $21,63 \%$, mientras que los programas a distancia tan solo del $1,04 \%$, lo que muestra una diferencia entre ambos tipos de programas de un 20,09\%; una de las diferencias más altas en lo que respecta a la flexibilidad disciplinar. Sin embargo, los programas a distancia presentan una mayor flexibilidad con respecto al componente de electivas libres, $11,78 \%$ frente a un $5,85 \%$ de promedio para los programas presenciales.

Pero la mayor diferencia relevante se encuentra entre los programas que pertenecen a instituciones oficiales $(40,31 \%)$ frente a los programas de instituciones privadas (15,76\%). La diferencia entre ambos tipos de programas es de un $24,55 \%$; la mayor diferencia que se presenta en esta clasificación. También es notoria la diferencia en flexibilidad disciplinar: mientras los programas de instituciones oficiales ofrecen en promedio un 32,49\% de créditos electivos, en las privadas es en promedio de 9,73\%; también es la mayor diferencia entre programas en la clasificación presentada. A continuación presentaremos los promedios de flexibilidad de los programas de filosofía, cruzando los datos de cada clasificación con respecto a las demás, para detectar diferencias importantes en los programas de acuerdo con características más específicas.

\section{Programas profesionales en filosofia}

De los 28 programas de estudios profesionales en filosofía los siguientes (tabla 4 y gráfica 9) son los promedios de flexibilidad de acuerdo con cada una de las demás clasificaciones enunciadas en la sección inicial $(\$ 1)$ :

En los programas de estudios profesionales en filosofía se encuentran diferencias considerables relacionadas con la flexibilidad de los programas según la clasificación presentada. Con respecto a la especificidad disciplinar, la flexibilidad de los programas exclusivamente filosóficos sigue siendo superior $(30,72 \%)$ con respecto a la flexibilidad de los programas que combinan filosofía con otras disciplinas (14,35\%). Es importante resaltar que la flexibilidad específicamente disciplinar también presenta una diferencia considerable, pues para los programas exclusivamente filosóficos presenta un $20,05 \%$ frente a un $10,86 \%$ de los programas que combinan filosofía con otras disciplinas. 
Tabla 4. Porcentaje promedio de flexibilidad en los programas profesionales en filosofía

\begin{tabular}{|l|l|c|r|r|c|}
\hline & & $\begin{array}{c}\text { N. } \\
\text { programas }\end{array}$ & $\begin{array}{c}\text { \% flexibilidad } \\
\text { disciplinar }\end{array}$ & $\begin{array}{c}\text { \% libre } \\
\text { elección }\end{array}$ & Flexibilidad \\
\hline \multirow{2}{*}{ Especificidad disciplinar } & Filosofía & 23 & $20,05 \%$ & $10,67 \%$ & $30,72 \%$ \\
\cline { 2 - 6 } & Filosofía y otro & 5 & $10,86 \%$ & $3,48 \%$ & $14,35 \%$ \\
\hline \multirow{2}{*}{ Registro ante el snies } & Registro calificado & 18 & $16,38 \%$ & $7,62 \%$ & $23,99 \%$ \\
\cline { 2 - 6 } & Registro alta calidad & 10 & $22,07 \%$ & $12,57 \%$ & $34,65 \%$ \\
\hline \multirow{2}{*}{ Modalidad } & Presencial & 26 & $19,67 \%$ & $9,14 \%$ & $28,80 \%$ \\
\hline \multirow{2}{*}{ Tipo de institución } & Distancia & 2 & $2,08 \%$ & $12,61 \%$ & $14,69 \%$ \\
\hline & Oficial & 11 & $28,44 \%$ & $15,16 \%$ & $43,60 \%$ \\
\hline & Privada & 17 & $11,92 \%$ & $5,65 \%$ & $17,57 \%$ \\
\hline
\end{tabular}

Gráfica 9. Porcentaje promedio de flexibilidad de los programas profesionales en filosofía

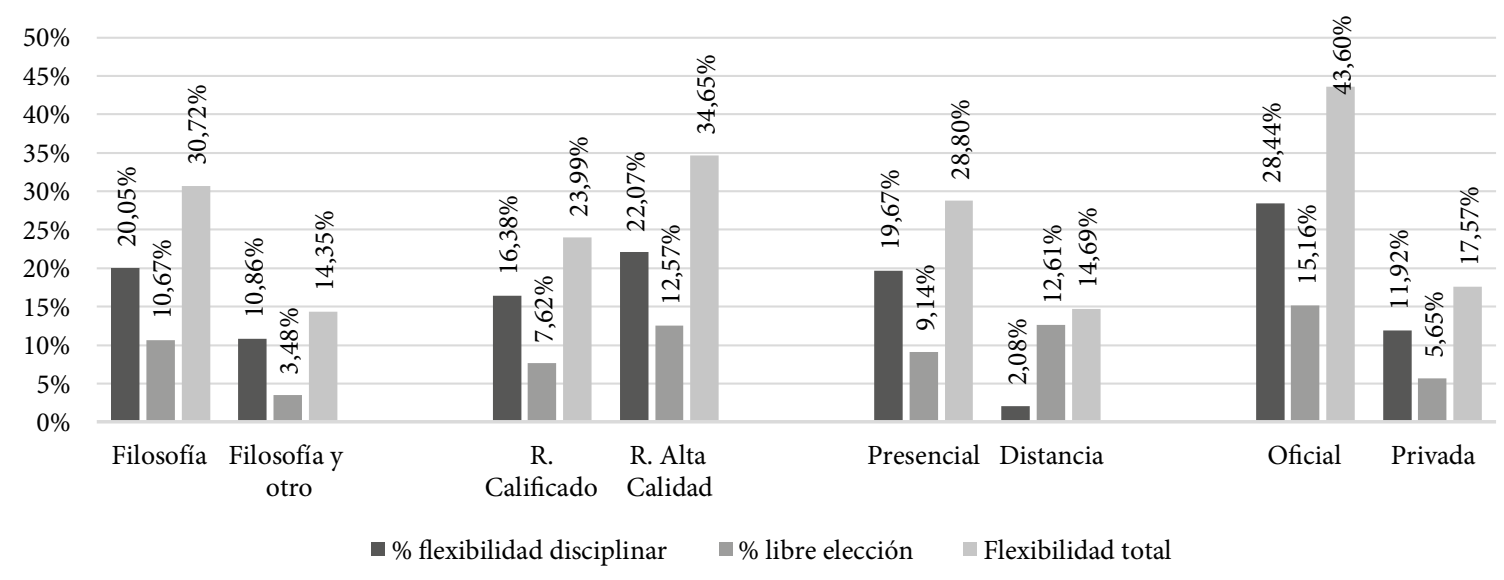

Fuente: Elaboración propia.

Con respecto al tipo de registro, sigue siendo mayor el promedio del porcentaje de flexibilidad los programas de alta calidad (34,65\%) frente a los programas que cuentan con registro calificado $(23,99 \%)$; de igual manera respondiendo a los criterios de evaluación de programas de alta calidad (CNA, 2013, pp. 30-31). Así mismo, hay una diferencia entre los programas de acuerdo con su modalidad: un $28,8 \%$ de media para los programas presenciales, y un $14,69 \%$ para los programas a distancia. Con respecto al tipo de institución, es considerablemente mayor la media del porcentaje de créditos en las instituciones públicas $(43,6 \%)$ con respecto al de las privadas (17,57\%).

Al comparar las diferencias con respecto al promedio general de los programas, podemos notar que las diferencias entre los programas de estudios profesionales de filosofía no presentan una diferencia considerable con respecto al promedio general.

\section{Licenciaturas en filosofía}

De los 26 programas de licenciaturas en filosofía las siguientes (tabla 5 y gráfica 10) son las medias de flexibilidad de acuerdo con cada una de las demás clasificaciones enunciadas en la sección inicial $(\$ 1)$ : 
Universidad Pedagógica Nacional

Facultad de Humanidades

Tabla 5. Porcentaje promedio de flexibilidad de las Licenciaturas en Filosofía

\begin{tabular}{|c|c|c|c|c|c|c|c|}
\hline & & $\begin{array}{c}\mathrm{N} .^{\circ} \mathrm{de} \\
\text { programas }\end{array}$ & $\begin{array}{c}\% \\
\text { flexibilidad } \\
\text { filosófica }\end{array}$ & $\begin{array}{c}\% \\
\text { flexibilidad } \\
\text { pedagógica }\end{array}$ & $\begin{array}{c}\% \\
\text { flexibilidad } \\
\text { disciplinar }\end{array}$ & $\begin{array}{l}\text { \% libre } \\
\text { elección }\end{array}$ & Flexibilidad \\
\hline \multirow{2}{*}{$\begin{array}{l}\text { Especificidad } \\
\text { disciplinar }\end{array}$} & Filosofía & 15 & $14,10 \%$ & $8,80 \%$ & $22,90 \%$ & $5,56 \%$ & $28,46 \%$ \\
\hline & $\begin{array}{l}\text { Filosofía } \\
\text { y otro }\end{array}$ & 11 & $7,65 \%$ & $1,24 \%$ & $8,89 \%$ & $5,78 \%$ & $14,67 \%$ \\
\hline \multirow{2}{*}{$\begin{array}{l}\text { Registro ante } \\
\text { el snies }\end{array}$} & $\begin{array}{l}\text { Registro } \\
\text { calificado }\end{array}$ & 20 & $10,13 \%$ & $5,08 \%$ & $15,21 \%$ & $6,46 \%$ & $21,67 \%$ \\
\hline & $\begin{array}{l}\text { Registro alta } \\
\text { calidad }\end{array}$ & 6 & $15,52 \%$ & $7,33 \%$ & $22,85 \%$ & $2,96 \%$ & $25,81 \%$ \\
\hline \multirow{2}{*}{ Modalidad } & Presencial & 20 & $14,58 \%$ & $7,28 \%$ & $21,86 \%$ & $3,90 \%$ & $25,76 \%$ \\
\hline & Distancia & 6 & $0,69 \%$ & $0,00 \%$ & $0,69 \%$ & $11,50 \%$ & $12,19 \%$ \\
\hline \multirow{2}{*}{$\begin{array}{l}\text { Tipo de } \\
\text { institución }\end{array}$} & Oficial & 10 & $18,60 \%$ & $13,70 \%$ & $32,30 \%$ & $4,40 \%$ & $36,70 \%$ \\
\hline & Privada & 16 & $6,86 \%$ & $0,54 \%$ & $7,40 \%$ & $6,43 \%$ & $13,83 \%$ \\
\hline
\end{tabular}

Gráfica 10. Porcentaje promedio de flexibilidad de las Licenciaturas en filosofía

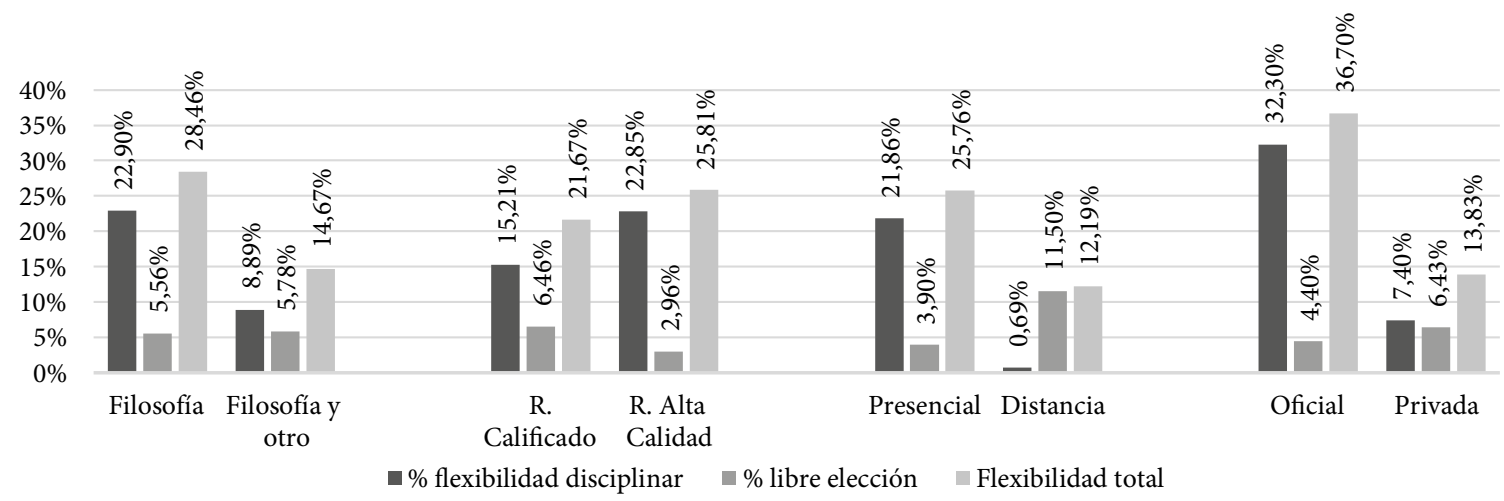

Fuente: Elaboración propia.

Con respecto a las licenciaturas en filosofía, las diferencias de acuerdo con las categorías enunciadas son similares. Las licenciaturas exclusivamente filosóficas presentan una mayor flexibilidad $(28,46 \%)$ que las que combinan filosofía con otras disciplinas $(14,67 \%)$. De igual manera, las licenciaturas con registro calificado $(21,67 \%)$ presentan un promedio de flexibilidad más bajo que las que tienen registro de alta calidad $(25,81 \%)$. Respecto de la modalidad de las licenciaturas, la tendencia es la misma que con los programas de estudios profesionales: las licenciaturas presenciales $(25,76 \%)$ son considerablemente más flexibles que las que son a distancia
$(12,19 \%)$. Es de resaltar la diferencia del porcentaje de flexibilidad del componente disciplinar: mientras que las presenciales tienen un promedio de $21,86 \%$ de créditos flexibles, los programas a distancia solamente ofrecen en promedio un $0,69 \%$ de créditos flexibles; esto evidencia que en las licenciaturas a distancia la flexibilidad de espacios académicos disciplinares es casi nula.

Sin embargo, la mayor diferencia detectada entre las licenciaturas se encuentra de acuerdo al tipo de institución: las instituciones oficiales presentan un promedio general de créditos flexibles de 36,70\% frente a un $13,83 \%$ de flexibilidad de los programas 
de instituciones privadas, lo que representa una diferencia de flexibilidad del 22,87\% de los planes de estudios. Así mismo, hay una diferencia relevante en la flexibilidad específicamente disciplinar de estos programas: mientras las licenciaturas de instituciones oficiales tienen un promedio de flexibilidad disciplinar del $32,30 \%$, las privadas tienen un promedio de $7,4 \%$, lo que representa una diferencia de flexibilidad disciplinar del $24,9 \%$ del plan de estudios.

\section{Conclusiones}

La principal conclusión que podemos obtener es que se presentan diferencias relevantes entre programas de filosofía, principalmente por tipo de institución: los programas de instituciones oficiales son, en general, más largos - especialmente las licenciaturas- y ofrecen una flexibilidad mucho mayor. Si la flexibilidad es un criterio importante de alta calidad para un programa (CNA, 2013, pp. 30-31), en este aspecto los programas de instituciones oficiales se encuentran en una situación ventajosa en relación a los programas de instituciones privadas. Con respecto a la propuesta de Lineamientos (MEN, 2014), en lo que se refiere a la duración de los programas, los programas que se verían más afectados serían los de instituciones privadas: nueve programas tendrían que aumentar su duración para cumplir con los diez semestres exigidos. En lo que respecta a la exigencia de que las licenciaturas sean presenciales, desaparecerían seis programas que se ofrecen a distancia.

Por último, es importante añadir que, si bien en el presente trabajo no podemos analizar con toda profundidad cada uno de los criterios de calidad que hemos examinado - duración, número de créditos y flexibilidad-, las diferencias que hemos presentado son indicadores importantes de diferencias de calidad bastante notables entre los programas. Es presumible que la mayoría de los programas que hemos estudiado aspiren a obtener el registro de alta calidad que otorga el CNA, y, en este sentido, esperamos que el presente estudio contribuya a identificar aspectos a mejorar en cada uno de los programas. Pero más allá de hacer un aporte en pro de alimentar la discusión para futuras reformas curriculares, para la apertura de nuevos programas, o para la postulación para obtener registro de alta calidad en algunos programas; nuestro propósitos con esta presentación del estado actual de los programas en filosofía en Colombia es hacer un aporte a la reflexión en torno al significado de la calidad misma de los programas académicos. Valga la pena aclarar que no creemos que exista un número créditos "exacto" o una duración "correcta" de un plan de estudios, ni que exista un porcentaje de flexibilidad "ideal" para cada programa; pero consideramos que hacer este comparativo permite examinar con detalle la enseñanza de la filosofía en educación superior en el contexto colombiano.

Parte de los resultados presentados aquí fueron estudiados por el comité de autoevaluación y de renovación del plan de estudios de la Licenciatura en Filosofía - del cual hicimos parte los autores de este trabajo-. Sirvió como insumo para discutir cuáles debían ser nuestras prioridades y qué cosas podrían ser prescindibles en el nuevo plan; así como qué cambios considerables se requerían - tal vez el más importante es el aumento de la flexibilidad del programa, de un $5 \%$ a un $22 \%$ - y qué elementos eran una fortaleza del plan anterior. También sirvió para identificar nuestras particularidades y para examinar la manera en la que el nuevo plan de estudios podría hacer frente a las necesidades propias del contexto en el que se encuentra nuestro programa y quienes hacen parte de él - tanto estudiantes como docentes y administrativos-. Por encima de cualquier otro criterio, es la eficacia para responder a estas necesidades lo que permite a largo plazo determinar la calidad de un programa.

\section{Trabajos citados}

Colegio Mayor de Nuestra Señora del Rosario. Filosofía. Plan de estudios. Disponible en: http://www.urosario.edu.co/cienciashumanas/Pregrados/Filosofia/ur/ Plan-de-Estudios/. Consultado el $1^{\circ}$ de abril de 2014.

Consejo Nacional de Acreditación. (2013). Lineamientos para la acreditación de programas de pregrado. Bogotá: Ministerio de Educación Nacional. Disponible en: http://www.cna.gov.co/1741/articles-186359_pregrado_2013.pdf. Consultado el 20 de abril de 2014. 
Corporación Universitaria Minuto de Dios. Estudios en Filosofía. Plan de estudios. Disponible en: http://www. uniminuto.edu/-/estudios-en-filosofia-en-bogota. Consultado el $1^{\circ}$ de abril de 2014.

Corporación Universitaria Minuto de Dios. Licenciatura en Filosofía. Plan de estudios. Disponible en: http:// www.uniminuto.edu/licenciatura-en-filosofiabogota-principal. Consultado el $1^{\circ}$ de abril de 2014.

Fundación Universidad Autónoma de Colombia. Filosofía. Plan de estudios. Disponible en: http://www. fuac.edu.co/index.php? $\bmod =422$. Consultado el $1^{\circ}$ de abril de 2014.

Fundación Universitaria Católica del Norte. Licenciatura en Filosofía y Educación Religiosa. Plan de estudios. Disponible en: http://www.ucn.edu.co/programasacademicos/Documents/pensum2012/Licenciatura filosofia.pdf. Consultado el $1^{\circ}$ de abril de 2014.

Fundación Universitaria Católica Lumen Gentium. Licenciatura en Filosofía y Ciencias Religiosas. Plan de estudios. Disponible en: http://www.unicatolica. edu.co/web/site/rutas/FCR.pdf. Consultado el $1^{\circ} \mathrm{de}$ abril de 2014.

Fundación Universitaria Luis Amigó. Filosofía. Plan de estudios. Disponible en: http://www.funlam.edu.co/ uploads/programas/pregrados/Filosofia-MED.pdf. Consultado el $1^{\circ}$ de abril de 2014.

Fundación Universitaria Luis Amigó. Licenciatura en Filosofía. Plan de estudios. Disponible en: http:// www.funlam.edu.co/uploads/programas/pregrados/ LicenciaturaFilosofia-MED.pdf. Consultado el $1^{\circ} \mathrm{de}$ abril de 2014.

Fundación Universitaria San Alfonso. Filosofía - Teología. Plan de estudios. Disponible en: http://www.fusa. edu.co/archivos/programa_filosofia_teologia.pdf. Consultado el $1^{\circ}$ de abril de 2014.

Fundación Universitaria San Alfonso. Licenciatura en Filosofía. Plan de estudios. Disponible en: http://www. fusa.edu.co/archivos/programa_licenciatura_filosofia.pdf. Consultado el $1^{\circ}$ de abril de 2014.

Ministerio de Educación Nacional (2014). Lineamientos de calidad para las licenciaturas en educación. Bogotá: Ministerio de Educación Nacional. Disponible en: http://www.mineducacion.gov.co/cvn/1665/articles-340962_recurso_1.pdf. Consultado el 25 de mayo de 2014.

Ministerio de Educación Nacional (2014) (2003). Decreto 2566 de septiembre 10. Por el cual se establecen las condiciones mínimas de calidad y demás requisi- tos para el ofrecimiento y desarrollo de programas académicos de educación superior y se dictan otras disposiciones. Bogotá: Ministerio de Educación Nacional. Disponible en: http://www.mineducacion.gov.co/1621/articles-86425_Archivo_pdf. pdf. Consultado el 26 de mayo de 2014. Pontificia Universidad Javeriana - Sede Cali. Licenciatura en Filosofía. Plan de estudios. Disponible en: http:// www.javerianacali.edu.co/sites/ujc/files/plan_de_ estudios_carrera_de_filosofia.pdf. Consultado el $1^{\circ}$ de abril de 2014.

Pontificia Universidad Javeriana. Filosofía. Plan de estudios. Disponible en: http://puj-portal.javeriana. edu.co/portal/page/portal/Facultad\%20de $\% 20$ Filosof\%EDa/plt_lic_filosofia/Plan\%20de\%20estudios. Consultado el $1^{\circ}$ de abril de 2014.

Pontificia Universidad Javeriana. Licenciatura en Filosofía. Plan de estudios. Disponible en: http://puj-portal. javeriana.edu.co/portal/page/portal/Facultad $\% 20$ de\%20Filosof\%EDa/plt_carrera_filosofia/Plan $\% 20$ de\%20estudios. Consultado el $1^{\circ}$ de abril de 2014.

República de Colombia. Ley 30 de 1992, por el cual se organiza el servicio público de la Educación Superior.

Universidad de Antioquia. Filosofía. Plan de estudios. Disponible en: http://www.udea.edu.co/portal/page/portal/SedesDependencias/Filosofia/C. estudiarInstituto/A.ProgramasPregrado. Consultado el $1^{\circ}$ de abril de 2014.

Universidad de Antioquia. Licenciatura en Filosofía. Plan de estudios. Disponible en: http://www.udea.edu.co/ portal/page/portal/SedesDependencias/Filosofia/C. estudiarInstituto/A.ProgramasPregrado. Consultado el $1^{\circ}$ de abril de 2014.

Universidad de Caldas. Filosofía y Letras. Plan de estudios. Disponible en: http://acad.ucaldas.edu. co/gestionacademica/planestudios/pensumver. asp $? \operatorname{cod}=412 \&$ cod_carrera $=025$. Consultado el $1^{\circ}$ de abril de 2014.

Universidad de Caldas. Licenciatura en Filosofía y Letras. Plan de estudios. Disponible en: http://acad.ucaldas. edu.co/gestionacademica/planestudios/pensumver. asp? cod_carrera $=213$. Consultado el $1^{\circ}$ de abril de 2014.

Universidad de Cartagena. Filosofía. Plan de estudios. Disponible en: http://www.unicartagena.edu. co/cienciashumanas/plandeestudiofilosofia.pdf. Consultado el $1^{\circ}$ de abril de 2014. 
Universidad de la Sabana. Filosofía. Plan de estudios. Disponible en: http://www.unisabana.edu.co/fileadmin/Documentos/Filosofia/plan_estudios_2010.pdf. Consultado el $1^{\circ}$ de abril de 2014.

Universidad de la Salle. Filosofía y Letras. Plan de estudios. Disponible en: http://unisalle.lasalle.edu.co/ media/docs/programas-academicos/pregrado/ malla-curricular-filosofia-y-letras.pdf. Consultado el $1^{\circ}$ de abril de 2014.

Universidad de los Andes. Filosofía. Plan de estudios. Disponible en: http://filosofia.uniandes.edu. co/index.php/programas/pregrado/descripcion. Consultado el $1^{\circ}$ de abril de 2014.

Universidad de Nariño. Licenciatura en Filosofía y Letras. Plan de estudios. Disponible en: http://pregrado.udenar.edu.co/?p=144. Consultado el $1^{\circ}$ de abril de 2014 .

Universidad de Pamplona. Filosofía. Plan de estudios. Disponible en: http://www.unipamplona.edu.co/ unipamplona/portalIG/home_1/recursos/facultades/artes/26052009/filosofia.jsp. Consultado el $1^{\circ}$ de abril de 2014.

Universidad de San Buenaventura. Licenciatura en Filosofía. Plan de estudios. Disponible en: http://www. usbbog.edu.co/index.php/programas-academicos/ carreras-profesionales/facultad-de-filosofia/licenciatura-en-filosofia/plan-de-estudios. Consultado el $1^{\circ}$ de abril de 2014.

Universidad del Atlántico. Filosofía. Plan de estudios. Disponible en: http://www.uniatlantico.edu.co/uatlantico/docencia/ciencias-humanas/programas/filosofia. Consultado el $1^{\circ}$ de abril de 2014.

Universidad del Cauca. Filosofía. Plan de estudios. Disponible en: http://www.unicauca.edu.co/versionP/oferta-academica/programas-de-pregrado/ filosofia/plan-de-estudios. Consultado el $1^{\circ}$ de abril de 2014.

Universidad del Quindío. Filosofía. Plan de estudios. Disponible en: http://portal.uniquindio.edu.co/fac/ humanas/index.php?option $=$ com_content\&view $=\mathrm{a}$ rticle\&id $=268 \&$ Itemid $=2$. Consultado el $1^{\circ}$ de abril de 2014.

Universidad del Valle. Licenciatura en Filosofía. Plan de estudios. Disponible en: http://filosofia.univalle.edu. co/Licenciatura_Filosofia/estructura_curricular. html. Consultado el $1^{\circ}$ de abril de 2014.

Universidad del Valle. Profesional en Filosofía. Plan de estudios. Disponible en: http://filosofia.univalle.edu.
co/Profesional_Filosofia/estructura_curricular.html. Consultado el $1^{\circ}$ de abril de 2014.

Universidad El Bosque. Filosofía. Plan de estudios. Disponible en: http://www.uelbosque.edu.co/programas_academicos/pregrado/filosofia. Consultado el $1^{\circ}$ de abril de 2014.

Universidad Externado de Colombia. Filosofía. Plan de estudios. Disponible en: http://190.7.110.123/pdf/1_ facultadCienciasSocialesHumanas/2012/10/filosofia. pdf. Consultado el $1^{\circ}$ de abril de 2014.

Universidad Industrial de Santander. Filosofía. Plan de estudios. Disponible en: http://www.uis.edu.co/ webUIS/es/academia/facultades/cienciasHumanas/ escuelas/filosofia/programasAcademicos/filosofia/ planEstudios.html. Consultado el $1^{\circ}$ de abril de 2014.

Universidad La Gran Colombia. Licenciatura en Filosofía e Historia. Plan de estudios. Disponible en: http:// www.ugc.edu.co/webmaster/cienciaseducacion/ documentos/Malla\%20filosofia.pdf. Consultado el $1^{\circ}$ de abril de 2014.

Universidad Libre. Filosofía. Plan de estudios. Disponible en: http://www.unilibre.edu.co/Filosofia/programa/ estructura-curricular1. Consultado el $1^{\circ} \mathrm{de}$ abril de 2014.

Universidad Nacional Abierta y a Distancia. Filosofía. Plan de estudios. Disponible en: http://www.unad. edu.co/estudios/index.php/filosofia/plan-de-estudios. Consultado el $1^{\circ}$ de abril de 2014.

Universidad Nacional Abierta y a Distancia. Licenciatura en Filosofía. Plan de estudios. Disponible en: http:// estudios.unad.edu.co/images/ecedu/archivosEscuela/Plan_de_estudios.pdf. Consultado el $1^{\circ}$ de abril de 2014.

Universidad Nacional de Colombia. Filosofía. Plan de estudios. Disponible en: http://www.humanas.unal. edu.co/filosofia/programas-curriculares/pregrado/. Consultado el $1^{\circ}$ de abril de 2014.

Universidad Pedagógica Nacional. Licenciatura en Filosofía. Plan de estudios. Disponible en: http://www.pedagogica.edu.co/admin/UserFiles/1257642595malla_ curricular_licenciatura_filosofa.pdf. Consultado el $1^{\circ}$ de abril de 2014.

Universidad Pedagógica y Tecnológica de Colombia. Licenciatura en Filosofía. Plan de estudios. Disponible en: http://www.uptc.edu.co/. Consultado el $1^{\circ} \mathrm{de}$ abril de 2014.

Universidad Pontificia Bolivariana. Filosofía y Letras. Plan de estudios. Disponible en: http://www.upb. 
edu.co/portal/page?_pageid=1054. 32439378\&_ $\mathrm{dad}=$ portal\&_schema $=$ PORTAL. Consultado el $1^{\circ}$ de abril de 2014.

Universidad Pontificia Bolivariana. Filosofía. Plan de estudios. Disponible en: http://www.upb. edu.co/pls/portal/docs/PAGE/GPV2_UPB_ MEDELLIN/PGV2_M030_PREGRADOS/PGV2_ M030080040_FILOSOF\%CDA/PROYECTO $\% 20$ EDUCATIVO\%20DEL\%20PROGRAMA\%20 -PEP-\%20FILOSOFIA.PDF. Consultado el $1^{\circ} \mathrm{de}$ abril de 2014.

Universidad Pontificia Bolivariana. Licenciatura en Filosofía y Letras. Plan de estudios. Disponible en: http://www.upb.edu.co/portal/page?_pageid=1054. 32439378\&_dad=portal\&_schema=PORTAL. Consultado el $1^{\circ}$ de abril de 2014.

Universidad Santo Tomas. Licenciatura en Filosofía y Educación Religiosa. Plan de estudios. Disponible en: http://www.ustadistancia.edu.co/images/stories/PLAN_DE_ESTUDIOS_FIL_EDUCACION_ RELIGIOSA.pdf. Consultado el $1^{\circ}$ de abril de 2014.

Universidad Santo Tomas. Licenciatura en Filosofía y Lengua Castellana. Plan de estudios. Disponible en: http://filosofia.usta.edu.co/images/stories/dependencia/pregrado/documentos/pensum_v2.pdf. Consultado el $1^{\circ}$ de abril de 2014.

Universidad Santo Tomas. Licenciatura en Filosofía. Ética $y$ Valores Humanos. Plan de estudios. Disponible en: http://www.ustadistancia.edu.co/images/stories/ PLAN_DE_ESTUDIOS_FIL_ETICA_Y_VALORES. pdf. Consultado el $1^{\circ}$ de abril de 2014.
Universidad Santo Tomas. Licenciatura en Filosofía. Pensamiento Político y Económico. Plan de estudios. Disponible en: http://www.ustadistancia.edu. co/images/stories/PLAN_DE_ESTUDIOS_FIL_ PENSAMIENTO_POLITICO_Y_ECONOMICO. pdf. Consultado el $1^{\circ}$ de abril de 2014.

Universidad Sergio Arboleda. Filosofía y Humanidades. Plan de estudios. Disponible en: http://www.usergioarboleda.edu.co/filosofia/FILOSOF\%C3\%8DA\%20 ANTIGUO/pe-filosofia-humanidades-2011.pdf. Consultado el $1^{\circ}$ de abril de 2014.

Universidad Sergio Arboleda. Licenciatura en Filosofía y Humanidades. Plan de estudios. Disponible en: http://www.usergioarboleda.edu.co/filosofia/ FILOSOF\%C3\%8DA\%20ANTIGUO/pe-licenciatura-humanidades-2011.pdf. Consultado el $1^{\circ} \mathrm{de}$ abril de 2014.

Universidad Tecnológica de Pereira. Licenciatura en Filosofía. Plan de estudios. Disponible en: http:// media.utp.edu.co/planeacion/archivos/documentos-de-interes-de-planeacion-academica/documentocondicionesiniciales-utp-julio-2009finall.pdf. Consultado el $1^{\circ}$ de abril de 2014.

Universitaria Agustiniana. Licenciatura en Filosofía. Plan de estudios. Disponible en: http://www.uniagustiniana.edu.co/index.php/plan-de-estudioslicenciatura-en-filosofia.html. Consultado el $1^{\circ}$ de abril de 2014. 